\title{
Modeling and Control of Torso Compass Gait Biped Robot with AI Controller
}

\author{
Abbas H.Miry \\ Electrical Engineering Dept. \\ AL-Mustansiriyah University \\ Baghdad, Iraq \\ Email: abbasmiry83@gmail.com
}

Abstract.

This work presents the mathematical model for a torso compass gait biped robot with three degrees of freedom (DOF) which is comprised of two legs and torso. Euler Lagrange method's is used to drive the dynamic equation of robot with computed control is used as a controller. The relative angles are used to simplify the robot equation and get the symmetry of the matrix. Convention controller uses critical sampling to find the value of $K_{P}$ and $\mathrm{K}_{\mathrm{v}}$ in computed controller, in this paper the Genetic optimization method is used to find the optimal value of $\mathrm{K}_{\mathrm{P}}$ and $\mathrm{K}_{\mathrm{v}}$ with suitable objective function which employ the error and overshoot to make the biped motion smooth as possible. To investigate the work of robot a Matlab 2013b is used and the result show success of modeling.

\section{Index Terms-Biped Robot, Computed Control, Lagrange. Genetic Algorithm.}

\section{Introduction}

In the past decades, there has been a growing interest in research with a view to reaching the functioning biped robot simulation of human displacement. Attention due to several reasons on the basis of the ability of the biped robot's movement of rough terrain environments or irregular forces does not move the wheeled robots. A number of possible applications in various fields are continuously emerging: the replacement of human beings in dangerous work such as rescue operations or restore the movement of persons with disabilities such as dynamically controlled prosthetics. Search has been relatively limited because of the complexity of the dynamics of the legs of a robot that grows with the number of links and degrees of freedom [1].

Methodology of investigation and dynamic of the so called the compass gait walking conduct intensive study. There are some important advantages of the compass gait model. First, this form of simplicity, the dynamics can be the most difficult analytically. Second, unlike most of the dynamic which traffic robots large curved compass presented a model is generally assumed points gait feet easily control foot placement in rugged terrain. Thirdly, because of its simplicity and this model can be easily extended to the existence of differences in systematic analysis [2].

\section{II.Compass-Gait Biped Robot}

Our work tries to modeling and controls the simple biped robot planar robot (shown in the figure. 1). [ $\left.\mathrm{q}_{1} \mathrm{q}_{2} \mathrm{q}_{3}\right]$ are joint angle, to simplify the modeling the relative angles $\left[\begin{array}{lll}\mathrm{Q}_{1} & \mathrm{Q}_{2} & \mathrm{Q}_{3}\end{array}\right]$ are used. The gait consists of support or the dance stage, dual support, which is the phase of immediate transition. During the stage of the individual support automated behaves like an inverted the pendulum double room with its support point resembles a point of suspension of the pendulum. During the stage of the dual support and the support of one of his legs. Use one of the natural equations and dynamic judgment during stage one support that separates the nonlinear dynamic restriction modes [2]. 


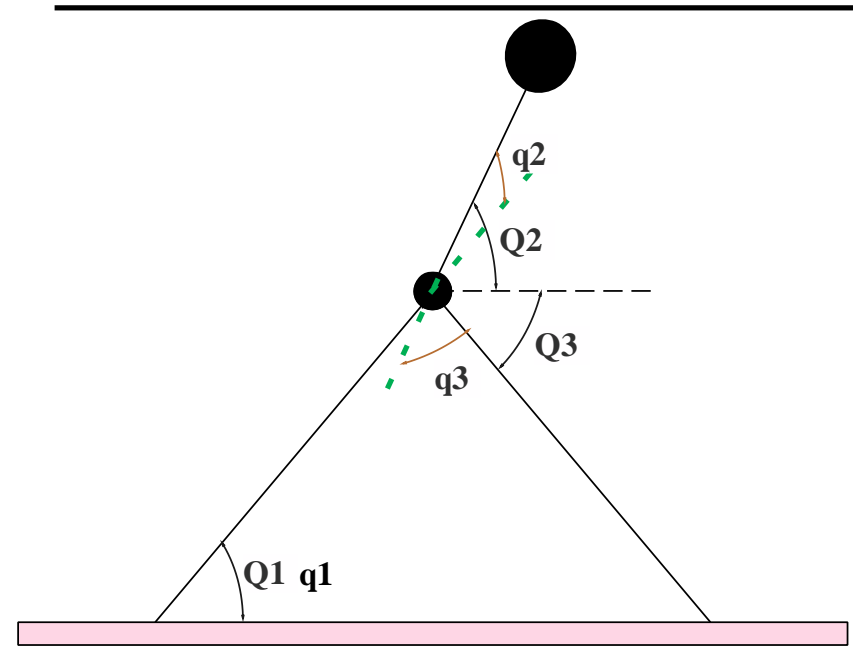

Fig.1 Compass-Gait Biped Robot Model

The governing equations of motion are derived using the Euler Lagrange approach as

$M(\theta) \ddot{\theta}+H(\theta, \dot{\theta})=\tau$

$H(\theta, \hat{\theta})=C(\theta, \dot{\theta}) \dot{\theta}+G(\theta)$

The parameters of the biped model are shown as follows [3]:

$M(\theta) \quad:[3 \times 3]$ inertia matrix of links.

$\theta \quad:[3 \times 1]$ Relative angle.

$C(q, \dot{q}):[3 \times 3]$ Coriolis torques.

$G_{q}(q):[3 \times 1]$ Gravitational torques.

$\tau \quad:$ [3x1] Joint Torque.

\section{Robot Dynamic}

Dynamic equations for robot manipulation in closed session a form that can be obtained by using the Lagrange equations. It is one of the more common approach used in the robot dynamic model in closed session. Lagrange equations of the movement can be used with the analysis of the growing number of joints in the robot [3].

The dynamic of biped robot is drives with single leg support phase considering in our work.

During the only station biped support stage had been submitted on the surface of the (support equality) carrying the weight of the biped other station in air. The Lagrange equation can be written in the form of [3]. $\frac{d}{d t}\left(\frac{d L}{d \theta_{i}}\right)-\frac{d L}{d \theta_{i}}=\tau_{i}$

$L=K-P$

(4)

Where

$K$ : Kinetic energy, $P$ : Potential energy

$M_{11} \ddot{\theta}_{1}+M_{12} \ddot{\theta}_{2}+M_{13} \ddot{\theta}_{3}+C_{11}(\theta, \dot{\theta}) \dot{\theta}_{1}+$ $C_{12}(\theta, \hat{\theta}) \dot{\theta}_{2}+C_{13}(\theta, \dot{\theta}) \dot{\theta}_{3}+G_{1}(\theta)=\tau_{1}$

(5)

$M_{21} \ddot{\theta}_{1}+M_{22} \ddot{\theta_{2}}+M_{23} \ddot{\theta}_{3}+C_{21}(\theta, \dot{\theta}) \dot{\theta}_{1}+$ $C_{22}(\theta, \hat{\theta}) \dot{\theta}_{2}+C_{23}(\theta, \dot{\theta}) \dot{\theta}_{3}+G_{12}(\theta)=\tau_{2}$

(6)

$M_{31} \ddot{\theta_{1}}+M_{32} \ddot{\theta_{2}}+M_{33} \ddot{\theta_{3}}+C_{31}(\theta, \dot{\theta}) \dot{\theta}_{1}+$ $C_{32}(\theta, \dot{\theta}) \dot{\theta}_{2}+C_{33}(\theta, \dot{\theta}) \dot{\theta}_{3}+G_{3}(\theta)=\tau_{3}$

Where

$$
\begin{aligned}
& M_{11}=I_{1}+m_{1} d_{1}^{2}+m_{2} l_{1}^{2}+m_{3} l_{1}^{2} \\
& M_{12}=l_{1} d_{2} m_{2} \cos \left(\theta_{1}-\theta_{2}\right) \\
& M_{13}=-l_{1} d_{3} m_{3} \cos \left(\theta_{1}+\theta_{3}\right) \\
& M_{21}=M_{12} \\
& M_{22}=I_{2}+m_{2} d_{2}^{2} \\
& M_{23}=0, M_{31}=M_{13}, M_{32}=M_{23} \\
& M_{33}=I_{3}+m_{3} d_{3}^{2}, C_{11}=0 \\
& C_{12}=l_{1} d_{2} m_{2} \sin \left(\theta_{1}-\theta_{2}\right) \dot{\theta}_{2} \\
& C_{13}=l_{1} d_{3} m_{3} \sin \left(\theta_{1}+\theta_{3}\right) \dot{\theta}_{3} \\
& C_{21}=-l_{1} d_{2} m_{2} \sin \left(\theta_{1}-\theta_{2}\right) \dot{\theta}_{1} \\
& C_{22}=0, C_{23}=0 \\
& C_{31}=l_{1} d_{3} m_{3} \sin \left(\theta_{1}+\theta_{3}\right) \dot{\theta}_{1} \\
& C_{32}=0, C_{33}=0 \\
& G_{1}=g\left(m_{1} d_{1}+m_{2} l_{2}+m_{3} l_{1}\right) \cos \left(\theta_{1}\right) \\
& G_{2}=g m_{2} d_{2} \cos \left(\theta_{2}\right) \\
& G_{3}=-g m_{3} d_{3} \cos \left(\theta_{3}\right) \\
& l_{i}: \text { Link Length } \\
& d_{i}: \text { Center of mass distance } \\
& I_{i}: \text { Moment of Inertia. }
\end{aligned}
$$

Table 1: Parameters of biped robot

\begin{tabular}{|c|c|c|c|c|}
\hline Link & $\begin{array}{c}\text { Mass } \\
(\mathrm{kg})\end{array}$ & $\begin{array}{c}\text { Link } \\
\text { Length(m) }\end{array}$ & $\begin{array}{c}\text { Center of } \\
\text { mass } \\
\text { distance }\end{array}$ & $\begin{array}{c}\text { Moment } \\
\text { of } \\
\text { Inertia }\end{array}$ \\
\hline 1 & 1.3 & 1 & 0.5 & 1.3 \\
\hline 2 & 0.3 & 0.25 & 0.125 & 0.4 \\
\hline 3 & 1.3 & 1 & 0.5 & 1.3 \\
\hline
\end{tabular}

\section{Computed Torque Control}

The computed torque (also called inverse dynamics) technique is a special application of 
feedback linearization of nonlinear systems. The computed torque controller is utilized to linearize the nonlinear equation of robot motion by cancellation of some, or all, nonlinear terms. For this purpose, the dynamic model of the robot is exploited [4].

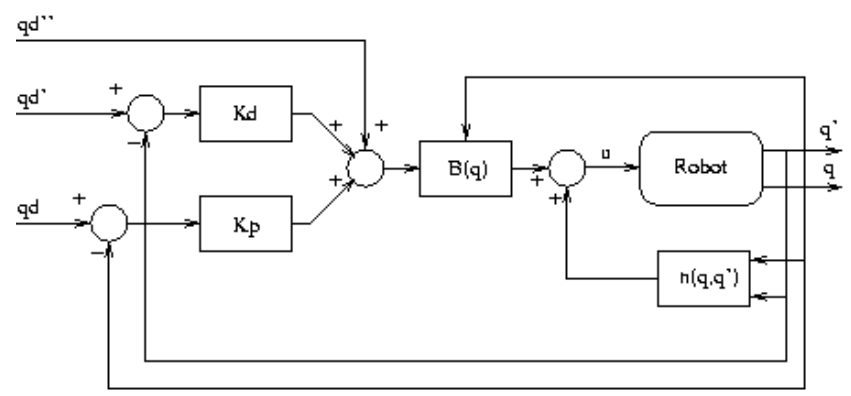

Fig.2 Computed torque control scheme

The computed torque equation is:

$T_{\theta}=M_{q}(\theta) u+N(\theta, \dot{\theta})$

The control signal $u$ is set as PD control law:

$u=\ddot{\theta}_{d}+K_{p} e+K_{v} \dot{e}$

$e=\theta-\theta_{d}$

$\dot{e}=\dot{\theta}-\dot{\theta}_{d}$

$\ddot{e}=\ddot{\theta}-\ddot{\theta}_{d}$

The closed-loop equation for the error tracking is [3]:

$\hat{\theta}_{d}+K_{p} \theta+K_{v} \dot{\theta}=0$

\section{Controller Design}

In this section the value of PD parameters are determined.The overall robot arm input is [5]

$T_{\theta}=M(\theta)\left(\ddot{\theta}_{d}+K_{v} \dot{e}+K_{p} e\right)+N(\theta, \dot{\theta})$

The error dynamics for closed loop are

$\ddot{e}+K_{v} \dot{e}+K_{p} e=0$

It can be rewritten in state-space form [6]

$\frac{d}{d t}\left[\begin{array}{l}e \\ \dot{e}\end{array}\right]=\left[\begin{array}{cc}0 & I \\ -K_{p} & -K_{v}\end{array}\right]\left[\begin{array}{l}e \\ \dot{e}\end{array}\right]$

The closed loop characteristic polynomial is

$\Delta_{c}(s)=\left|s^{2} I+K_{v} s+K_{p}\right|$

The error system is asymptotically stable as long as $K_{p}$ and $K_{v}$ are all positive [7]. This value may be achieved by selecting the PD gains as

$K_{p}=w_{n}^{2}$

$K_{v}=2 w_{n} \xi$
Where $\xi$ desired damping ratio, $w_{n}$ natural frequency

It is not desirable that the robot overshoot, Therefore, the $K_{p}$ and $K_{v}$ usually selected for critical sampling $\xi=1$.

$K_{v}=2 \sqrt{K_{p}}$

Genetic algorithm (GA) is stochastic optimization method. It works with the population of the initial chromosome that regenerated through some operations such selection and crossover and mutation in number of generations until to reach the best solution well on maintaining the best individuals on the basis of evaluation criteria. In every generation chromosomes individually using the evaluation function and performance measures called fitness function [8].

The implementation of controller parameter tuning based Genetic algorithm begins with defines the representation of the chromosome. The chromosome contains six values which correspond to the gain of controller.
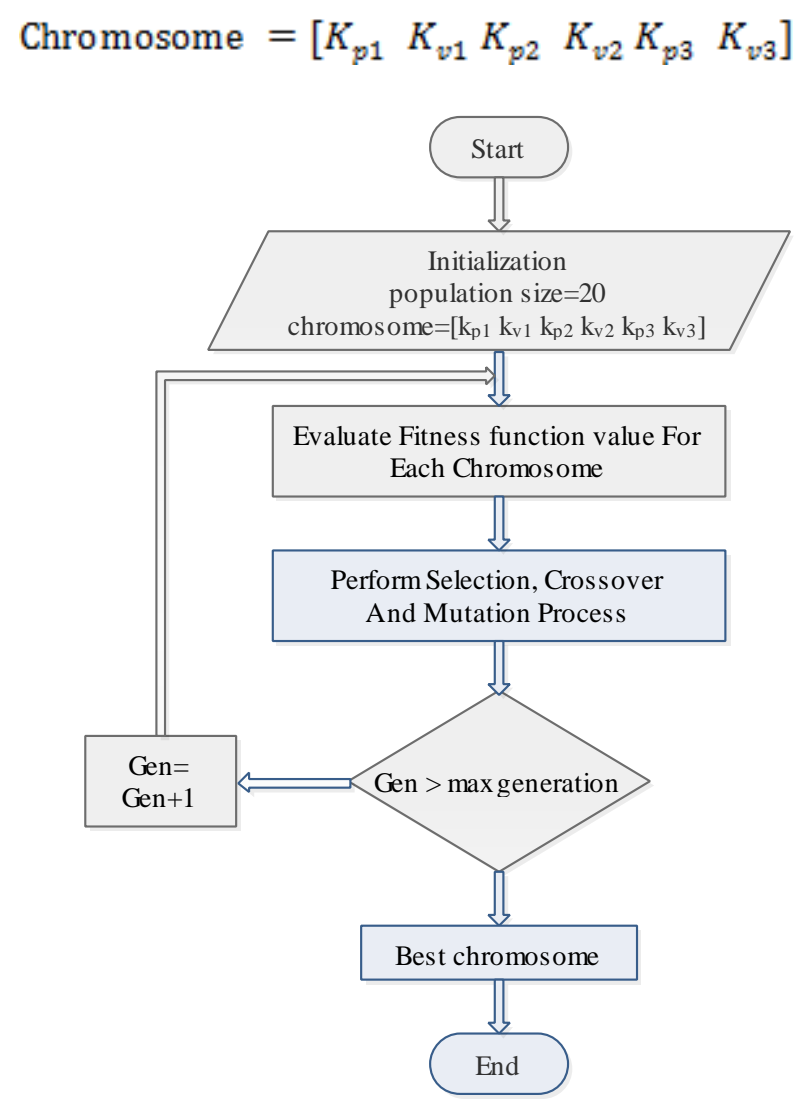

Fig.3 Flow chart of Genetic algorithm In this paper $K_{p}$ and $K_{v}$ values are determined using fitness function: 
fitness $=\sum|e|+{ }_{\max }\left|e_{1}\right|+{ }_{\max }\left|e_{2}\right|+$ $\max \left|e_{3}\right|+t_{\text {rise }}+t_{\text {settling }}$

The term $\max |e|$ is added to reduce the overshot in each joint, $t_{\text {rise }} t_{\text {settling }}$ are added to make the response faster.

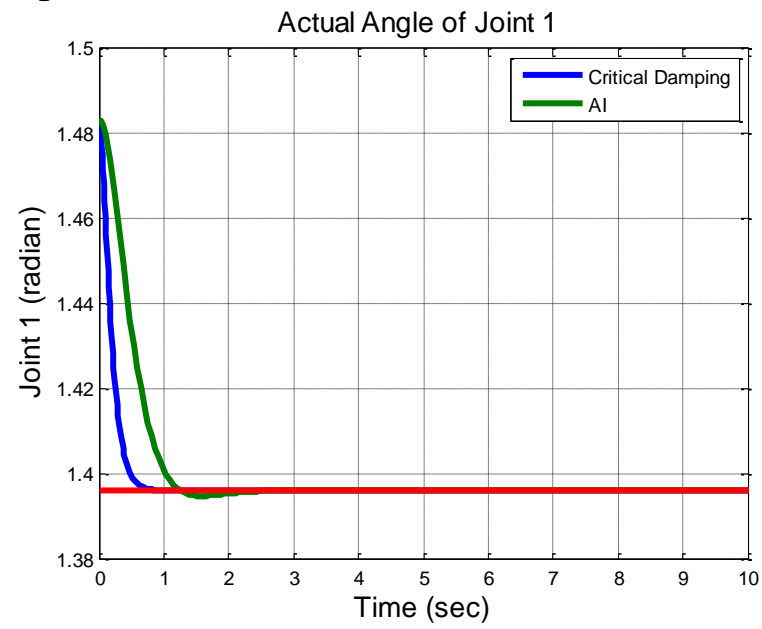

Fig.4 Actual angle of joint 1

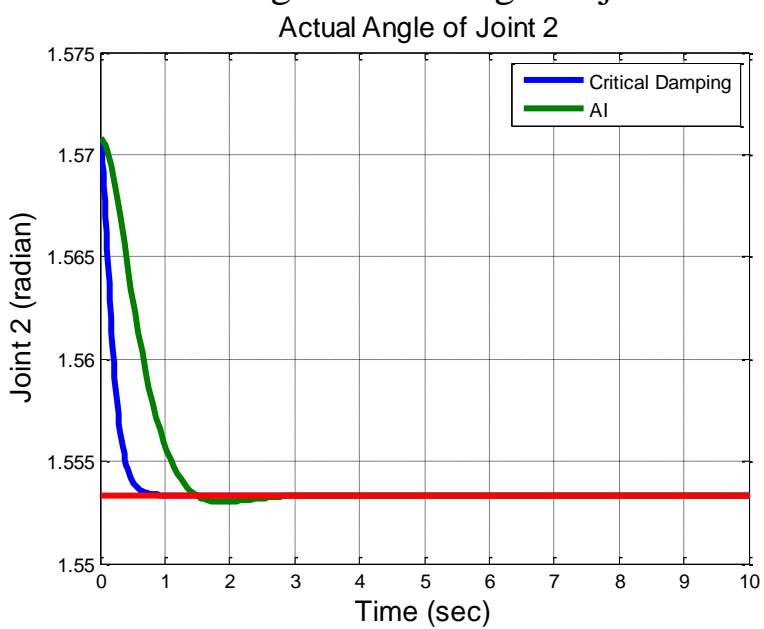

Fig.5 Actual angle of joint 2
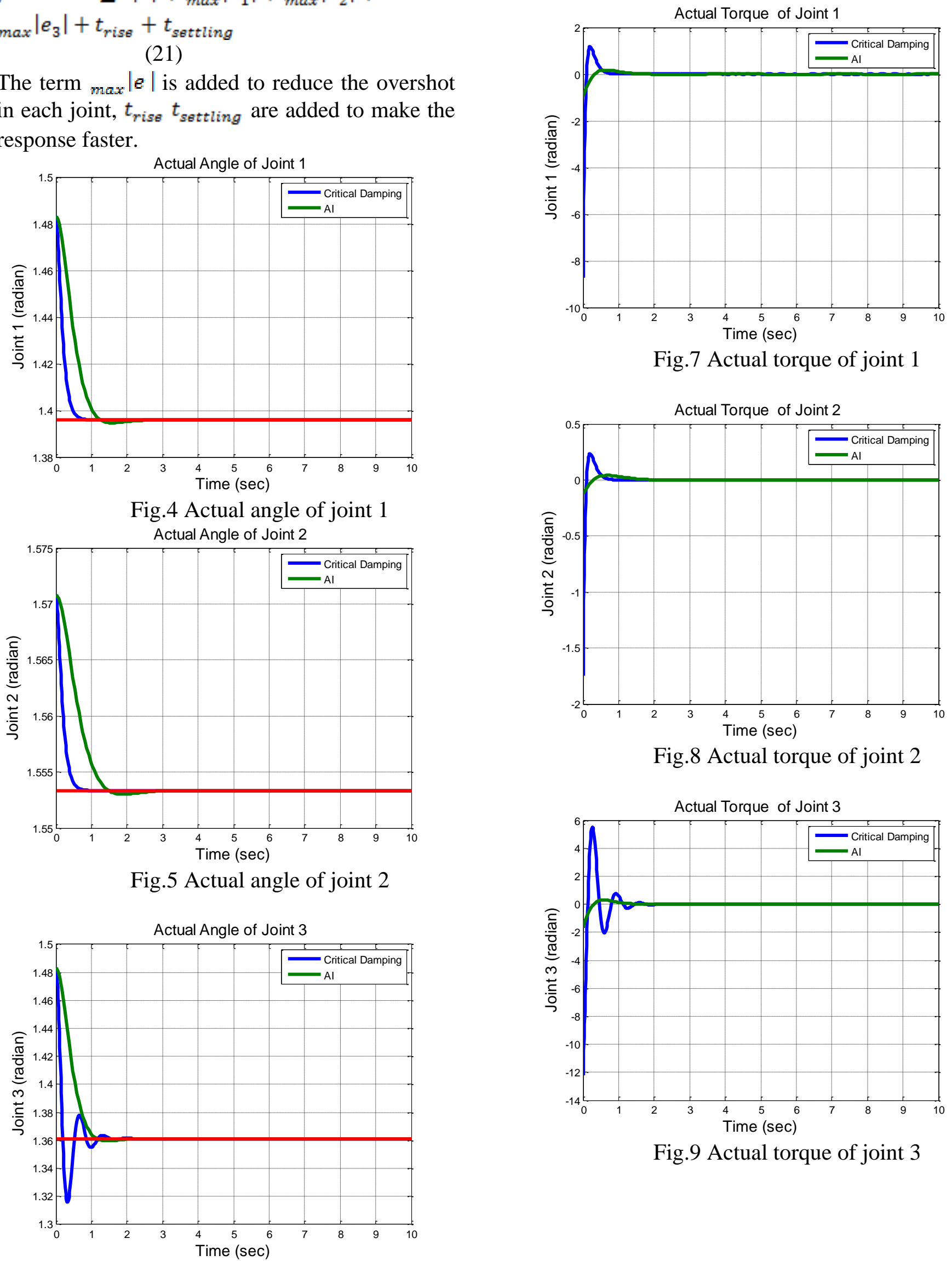

Fig.7 Actual torque of joint 1

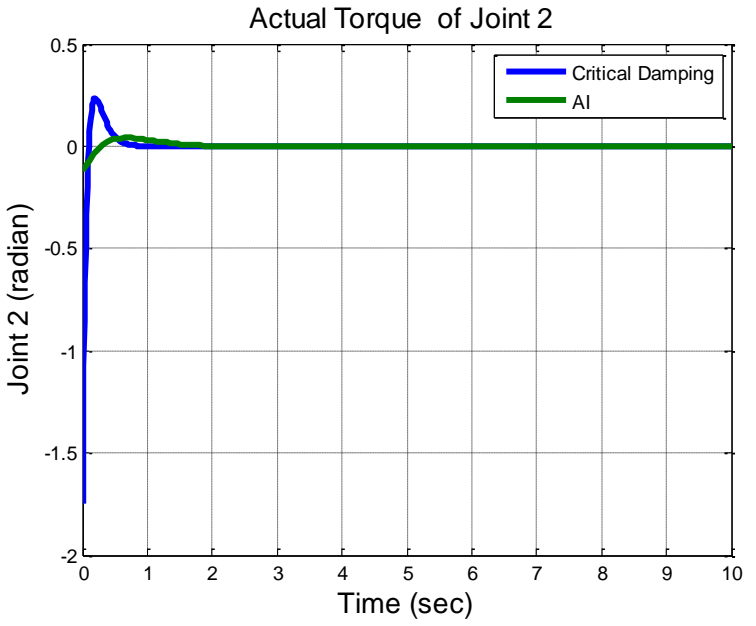

Fig.8 Actual torque of joint 2

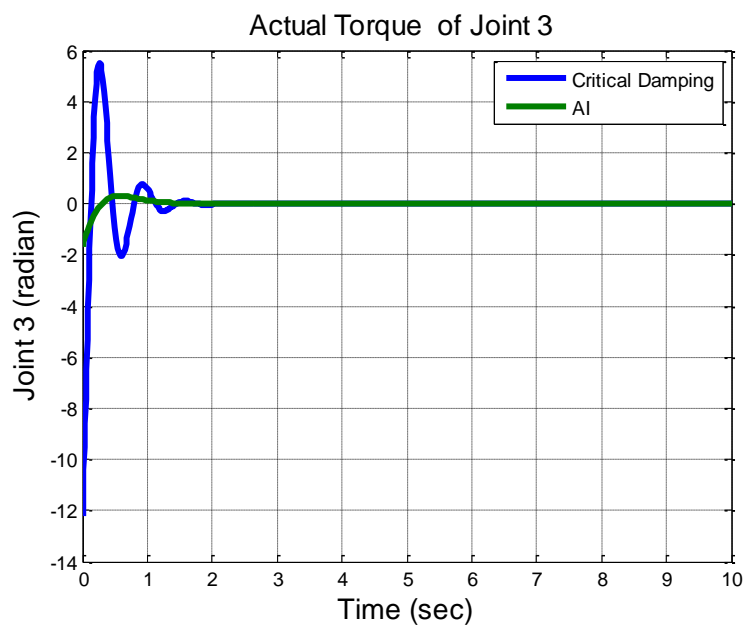

Fig.9 Actual torque of joint 3

Fig.6 Actual angle of joint 3 


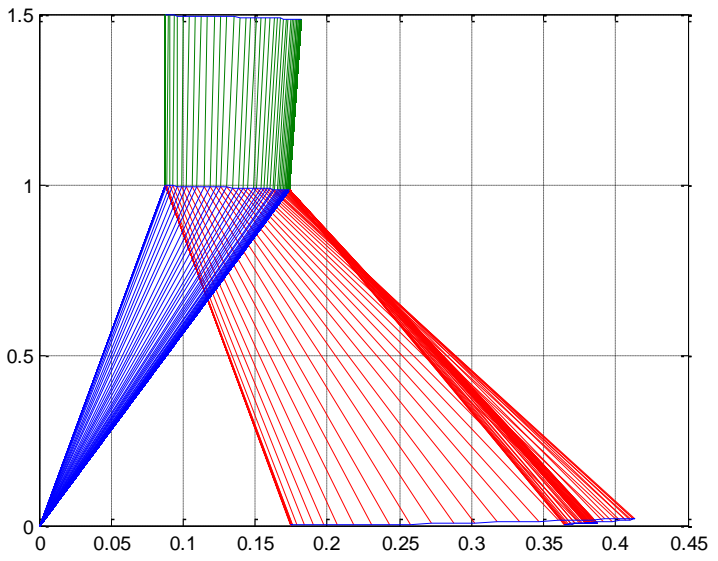

Fig.10 Motion of biped robot based Critical damping

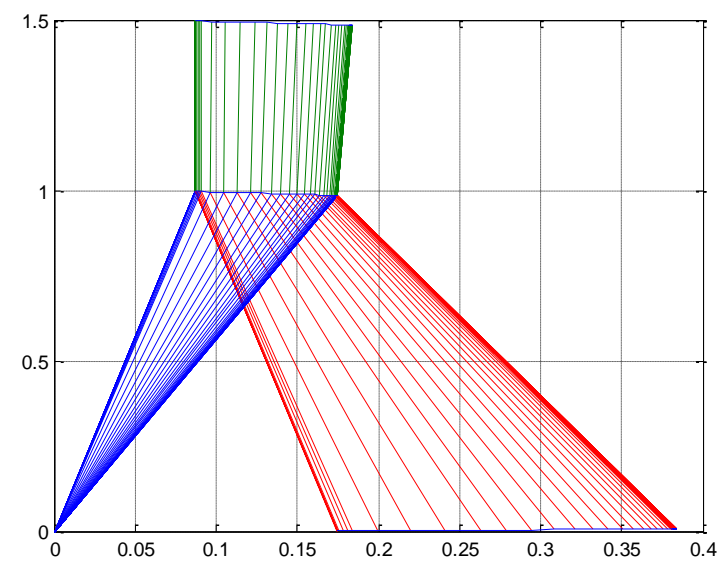

Fig.11 Motion of biped robot based AI

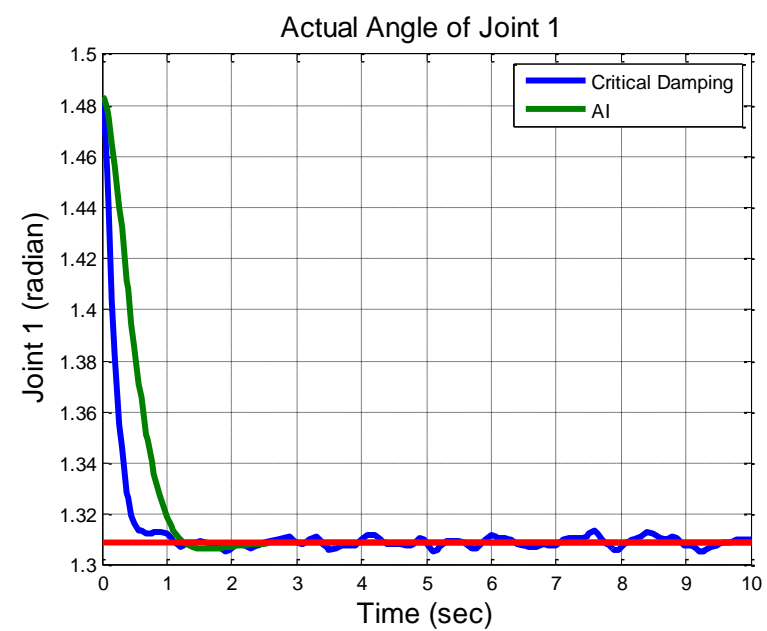

Fig.12 Actual torque of joint 1 with disturbance

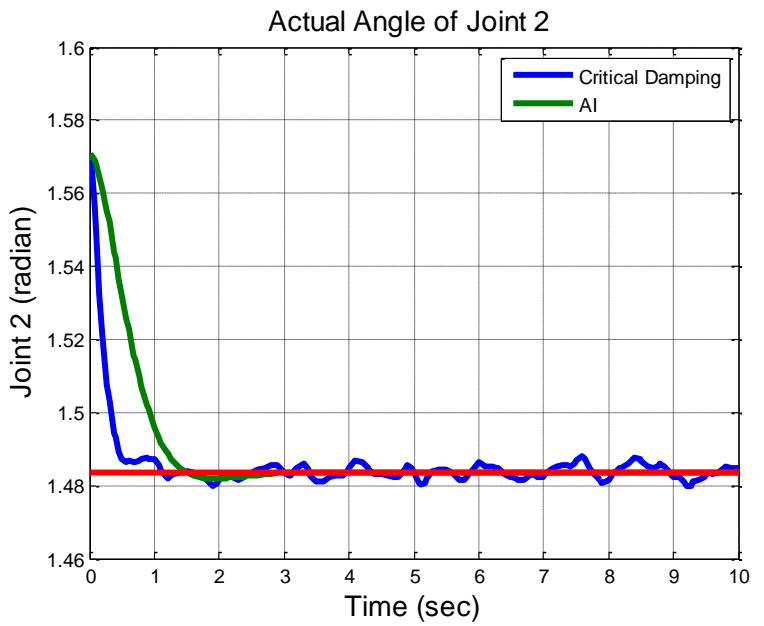

Fig.13 Actual torque of joint 2 with disturbance

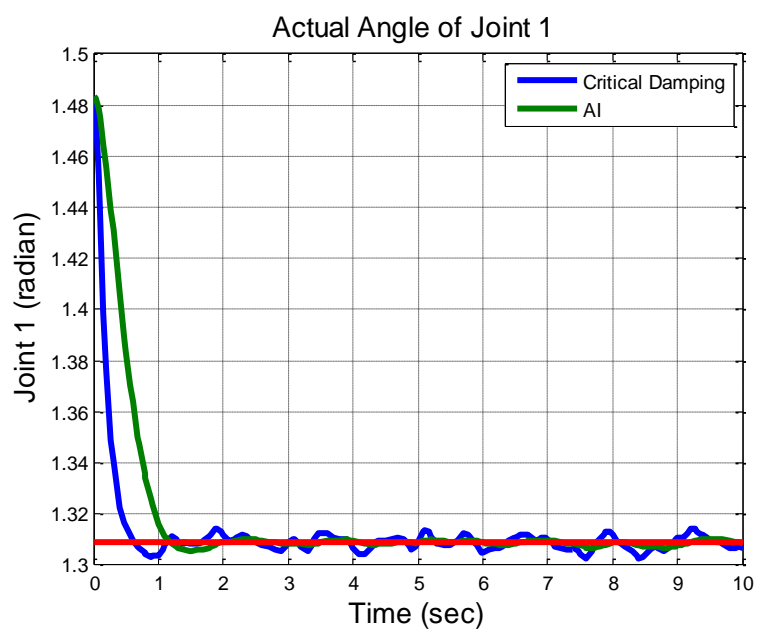

Fig.14 Actual torque of joint 1 with noise

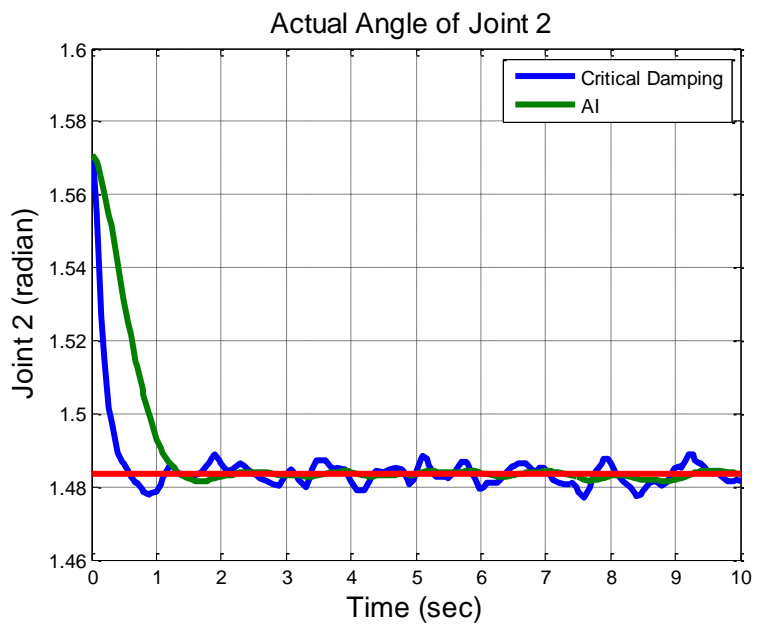

Fig.15 Actual torque of joint 2 with noise 


\section{Experimental Results}

The experimental result animates the biped walking on a horizontal plane using the Program MATLAB 2013b. The following figures show the actual angle, the actual torque of each joint for PD parameter of Critical sampling and Artificial Intelligent.

The performance of the various methods is evaluated by comparing the angle and torque response produced by three joints during the simulation, as shown in figures. On the basis of the results obtained in figures 4-11, AI Controller response is better than a critical controller due to good choosing the PD parameter using optimization also the overshot is eliminated.to test the robustness of AI controller the disturbance and noise are added to the system. figures 12-15 show the response of system ,from these figures it be noticed the AI system response is more robust than critical damping .

\section{Conclusion}

Our work provides a methodology for the derivation of the biped movement equations with three-link robot. In this paper the dynamic equation of Torso Compass Gait biped robot is derived using Lagrange's equation method with modeling and control. conventional control is used critical sampling to choose PD parameter, genetic optimization is used in our work to find the optimal value of PD parameters with a fitness function implode the error and overshoot value, this choice makes the response of $\mathrm{AI}$ controller is better and more robust than conventional control as shown in result especially in joint three.

Matlab is used as a verification tool to simulate the motion with angles and torque response for each joint in order to explore the control of the movement and performance of biped robot using the mathematical model.

\section{Reference}

1. M. F.Lobo, J. Manuel, J. L.González ," Modeling a Biped Robot on Matlab/SimMechanics ", , International Conference on Electronics, Communications and Computing , 2013 , PP:203-206.
2. F. Iida and R. Tedrake," Minimalistic control of biped walking in rough terrain", Proceedings of the 2009 IEEE international conference on Robotics and Automation PP: 3246-3251.

3. N. Onn, M. Hussein, C.H.H. Tang, M.Z.M. Zain M. Mohamad and L. Ying, "Motion control of seven-link human bipedal model" ,Robotics, control and manufacturing technology; Manufacturing engineering, automatic control and robotics , pp: 15-22 .

4. A.Jahed , F.Piltan, H.Rezaie and B.Boroomand ," Design Computed Torque Controller with Parallel Fuzzy Inference System Compensator to Control of Robot Manipulator " I.J. Information Engineering and Electronic Business, issue: 3,pp: 66-77 ,2013.

5. J. Peng1 and Y. Liu," Adaptive Robust Quadratic Stabilization Tracking Control for Robotic System with Uncertainties and External Disturbances", Journal of Control Science and Engineering Vol. 2014, pp: 1-10.

6.S. Soltani and F. Piltan," Design Artificial Nonlinear Controller Based on Computed Torque like Controller with Tunable Gain", World Applied Sciences Journal Vol.14, No.9, pp: 13061312, 2011.

7. N. Munro,"Robot Manipulator Control Theory and Practice", Second Edition, Marcel Dekker, Inc, 2004.

8. F. S. Osorio, M. R. Heinen, "Applying Genetic Algorithms to Control Gait of Simulated Robots", Electronics, Robotics and Automotive Mechanics Conference, CERMA, pp. 500-505, 2007. 